\title{
What Clinical and Laboratory Parameters Distinguish Between Acute and Chronic Renal Failure?
}

\author{
Abubakr A Sanusi ${ }^{a}$, Fatiu A Arogundade ${ }^{a^{*}}$, Theresa G Ekwere ${ }^{a}$, Adewale Akinsola ${ }^{a}$ \\ a. Renal Unit, Obafemi Awolowo University Teaching Hospitals Complex, Ile-Ife, Nigeria
}

\begin{abstract}
Introduction: In developing countries, a large number of patients presenting acutely in renal failure are indeed cases of advanced chronic renal failure. In this study, we compared clinical and laboratory parameters between patients with acute renal failure (ARF) and chronic renal failure (CRF), to identify discriminatory features.
\end{abstract}

Patients and methods: The Renal Unit of Obafemi Awolowo University Teaching Hospitals Complex is a major referral center for renal disease in Nigeria. 20 patients with ARF and 22 patients with CRF (who had not had dialysis intervention) were recruited for the study at presentation. They had full evaluation including demography, history of duration of symptoms, blood pressure, volume of urine, and laboratory parameters: serum creatinine, urea, potassium, and packed cell volume (PCV). These parameters were compared using Mann Whitney U test for nonparametric data to determine statistical significance.

Results: There were no significant differences between the two groups regarding their (i) ages (ii) serum creatinine and (iii) PCV.

In contrast, statistically significant differences were obtained for (i) the mean duration of symptoms, which was longer in CRF patients, (ii) the mean 24 hour urine volume, which was larger in CRF patients, (iii) the mean systolic and diastolic blood pressures, both being significantly higher in CRF patients, (iv) and the mean serum urea level, which was higher in ARF patients.

Conclusion: It is concluded that the duration of symptoms, quantity of urine, blood pressure, and serum urea levels are distinguishing parameters between ARF and $\mathrm{CRF}$, while serum creatinine and PCV are not.

\footnotetext{
* Corresponding Author; Renal Unit, Obafemi Awolowo University Teaching Hospitals Complex, P.M.B. 5538, Ile-Ife, Nigeria. E mail: fatiu3@yahoo.com. Fax: (+234)362 30141
}

Key words: Acute Renal Failure, Chronic Renal Failure, Laboratory Parameters

\section{Introduction}

Published studies indicate that parameters such as anemia and serum creatinine, which were once widely held to distinguish between acute renal failure (ARF) and chronic renal failure (CRF), are not clearly discriminatory [1-3], and that a considerable proportion of ARF patients are anaemic at diagnosis [4]. Newer distinguishing features are emerging and these include assessment of carbamylated hemoglobin concentration [5] and fingernail creatinine values [6].

In developing countries, a large number of patients presenting acutely in renal failure are indeed cases of advanced chronic renal failure [3,7-9] who need to be clearly distinguished from acute renal failure patients, in order to channel the limited resources to those who would benefit most. In many of the centers, available investigations are limited to only serum urea, serum creatinine, packed cell volume (PCV) and serum electrolytes $(\mathrm{Na}, \mathrm{K}, \mathrm{HCO} 3, \mathrm{Cl})$, while parameters such as serum calcium, phosphate or alkaline phosphatase are not routinely available.

We evaluated our cases of ARF and CRF to determine which parameters could be used to distinguish between them in resource poor settings found in most developing countries.

\section{Patients and methods}

Forty two consecutive cases of renal failure that were managed in our center were studied.

Twenty patients were diagnosed and managed as ARF on the following grounds: a dramatic rise in serum urea and creatinine, normal sized kidneys on ultrasonography and recovery of renal function on follow up within 3 months (in those who survived). We excluded cases with obstructive uropathy, those with significant proteinuria or sugar in spot urine test with combur-9 strip and those with 
Table 1: Comparison of age, duration of illness, blood pressures and urine volume in acute and chronic renal failure groups

\begin{tabular}{llllll}
\hline Parameter & ARF & & CRF & \multicolumn{2}{c}{ P value } \\
\cline { 2 - 5 } & Range & Mean \pm SD & Range & Mean \pm SD & \\
\hline Age (years) & & $34.2 \pm 12.1$ & & $38.4 \pm 10.6$ & 0.07 \\
Duration of symptoms (days) & $2-14$ & $9.2 \pm 10$ & $7-150$ & $35 \pm 20$ & $<0.001$ \\
Systolic blood pressure (mmHg) & $70-200$ & $119.0 \pm 28$ & $130-260$ & $184.1 \pm 34$ & $<0.0001$ \\
Diastolic blood pressure (mmHg) & $50-110$ & $72.5 \pm 140$ & $90-150$ & $123.3 \pm 187$ & $<0.0001$ \\
Volume of urine (ml/d) & $0-1950$ & $363.0 \pm 516$ & $210-3800$ & $1236.1 \pm 126.1$ & 0.000 \\
\hline
\end{tabular}

Table 2: The differences between ARF and CRF patients in some pertinent laboratory data

\begin{tabular}{llllll}
\hline Parameter & ARF & & CRF & \multicolumn{1}{c}{ P value } \\
\cline { 2 - 4 } & Range & Mean \pm SD & Range & Mean \pm SD \\
\hline Serum creatinine $(\mu \mathrm{mol} / \mathrm{L})$ & $240-2177$ & $1029 \pm 501.1$ & $410-1719$ & $881.2 \pm 337.9$ & 0.14 \\
Serum urea $(\mathrm{mmol} / \mathrm{L})$ & $13.4-51$ & $28.8 \pm 8.6$ & $8.9-327$ & $19.1 \pm 5.9$ & 0.000 \\
Serum potassium $(\mathrm{mmol} / \mathrm{L})$ & $3.6-6.1$ & $4.5 \pm 0.83$ & $2.5-5.7$ & $4.17 \pm 0.7$ & 0.18 \\
Packed cell volume $(\%)$ & $11-36$ & $19.0 \pm 6.8$ & $11-35$ & $22.6 \pm 5.1$ & 0.14 \\
\hline
\end{tabular}

a defined cause of anemia such as glucose-6-phosphate dehydrogenase deficiency and hemoglobinopathies. Acute glomerulonephritis (GN) was excluded by the absence of haematuria and proteinuria.

Twenty two patients had CRF, characterised by a less dramatic rise in serum urea and creatinine, ultrasonographic evidence of shrunken kidneys, and failure of renal function to return to normal on follow-up, the majority being dialysis dependent.

Their history was noted particularly for symptoms indicative of renal disease, this was used to determine the duration of symptoms. The following clinical and laboratory parameters were noted, namely: age, gender, blood pressure, volume of urine per day, serum potassium, serum urea, serum creatinine, and PCV. Data was analysed using SPSS package version 9. Values were expressed as mean \pm SD. Mann Whitney U test for nonparametric data was used to compare the two groups.

\section{Results}

(Table 1) summarizes the differences between patients with ARF and CRF on the first clinical encounter (age, duration of symptoms, blood pressure and urine volume). There were 12 and 14 males in the ARF and CRF groups respectively while there were 8 females in each group. The age was not significantly different between the two groups.

The difference between the two groups in terms of the duration of symptoms was statistically significant $(\mathrm{P}<0.001)$, being longer in patients with $\mathrm{CRF}$ compared to ARF. Also, the systolic and diastolic blood pressure readings were significantly higher in CRF patients $(\mathrm{P}<0.001)$. The urine volume was significantly lower in ARF patients $(\mathrm{P}=0.00)$.

(Table 2) displays several pertinent laboratory investigations results in ARF and CRF patients. Differences in PCV and serum biochemical parameters including potassium and creatinine were not statistically significant between the two groups. However, serum urea levels were significantly higher in ARF patients $(\mathrm{P}=0.00)$.

\section{Discussion}

The distribution of ARF may vary with the type of hospital (community versus referral) and with geographic region. However, in general, pre-renal azotemia and ischemic acute tubular necrosis (ATN) occur as a continuum of the same pathophysiological process and together account for $75 \%$ of the cases of ARF.

ATN in intensive-care units can be attributed to sepsis in $35-50 \%$ of the cases. ATN after surgery accounts for $20-25 \%$ of all cases of hospital-acquired ARF; many of them have pre-renal causes. Acute contrast nephropathy is the third commonest cause of ATN in patients admitted to hospital [10].

Acute renal failure in our setting is usually due to sepsis, nephrotoxic injury and severe pre-renal insult [11-13]. In the majority of cases, the outcome is favourable if relevant facilities are available [14].

In the tropics it is common for patients with CRF to present in an acute way, either because patients only 
vaguely recollect a past history of renal disease or are oblivious to the symptoms and signs of disease, or because of rapid progression of the disease [3,6-8]. The limited financial resources against that background and the irreversibility of renal function in advanced chronic renal failure compel a clear distinction between ARF and CRF so that resources could be judiciously utilized for those that would benefit most.

It was believed that the presence of anemia in patients with renal failure is indicative of CRF [1]. This study and others as well [5] do not support this view, and it is felt that less emphasis should be placed on anemia as a discriminatory parameter.

Where it could be established, the duration of symptoms is a clear discriminatory parameter. Patients knowledge of symptoms and signs of renal disease has to improve if this very simple but important parameter (duration of symptoms) is to be effectively utilized.

Blood pressure levels seem to clearly distinguish between $\mathrm{ARF}$ and CRF. Hypertension is prominent in most cases of CRF and may reflect the underlying aetiology of the $\mathrm{CRF}$, and this underscores the values of a good history. Hypertension can be seen in ARF in the setting of acute GN, but this is generally a disease of childhood in our setting.

While oliguria was prominent among patients with ARF, a few of them (5 of the 22) were non-oliguric, passing between 700 and $1950 \mathrm{ml}$ of urine per day. Only six of the 22 patients with CRF passed urine volume below $500 \mathrm{ml} /$ day. Urine output at presentation is a useful discriminatory parameter, with patients of ARF being generally oliguric while those with CRF pass normal to excessive urine volumes.

It is expected to find that serum creatinine levels do not distinguish ARF from CRF. Serum creatinine can only be reliably used to assess renal function in patients with stable kidney function. In severe acute renal failure, for example, the glomerular filtration rate (GFR) is markedly reduced but there has not yet been time for creatinine to accumulate and for the serum creatinine level to reflect the degree of renal dysfunction [15]. Very high levels, (values greater than $1000 \mu \mathrm{mol} / \mathrm{l}$ ) are commonly encountered in ARF as well as CRF.

Although the serum urea level varies inversely with the GFR, it is generally less useful than the serum creatinine, because it can change independently of the GFR. The rate of urea production increases with a high protein diet and with enhanced tissue breakdown. By comparison, a low protein diet or liver disease can lower the urea without a change in GFR. Approximately 40 to 50 percent of the filtered urea is passively reabsorbed; thus, when volume depletion is associated with enhanced proximal sodium and water reabsorption there is a parallel increase in urea reabsorption. As a result, the urea will rise out of proportion to any change in GFR and therefore to any change in the serum creatinine. This elevation in the urea-to-creatinine ratio is one of the suggestive clinical signs of decreased renal perfusion (pre-renal disease) as the cause for ARF [16]. Davenport et al [4] observed that serum urea was lower in CRF patients than ARF patients, a difference they ascribed to the possible lower protein intake by the former. We also observed a statistically significant lower serum urea level in CRF patients compared to ARF patients, possibly also due to protracted illness in CRF with poorer appetite and grossly reduced protein intake.

Ultrasonographic parameters clearly distinguish ARF from CRF. Apart from the difference in bipolar and transverse diameters, we observed that the distinctiveness of the cortico-medullary differentiation (CMD) also distinguished between ARF and CRF, with CMD being indistinct in CRF.

\section{Conclusion}

It is therefore concluded that a good history aimed at determining the duration of symptoms will be very helpful in categorising renal failure. Urine output at presentation, the presence of hypertension, and serum urea level are also distinctive features useful in distinguishing between ARF and CRF. Less emphasis should be placed on the presence of anemia, serum creatinine and serum potassium levels as parameters for distinguishing ARF from CRF.

\section{References}

1. Kanis JA. Disorders of calcium metabolism. In: Neatherall DJ, Ledingham JGG, and Warrel DA, editors. Oxford textbook of medicine, Vol (1). $2^{\text {nd }}$ ed. New york: Oxford University Press; 1987. p. 10.15-10.52.

2. Del Greco F, Krumloysky PA. Role of the laboratory in management of acute and chronic renal failure. Ann Clin Lab Sci. 1981;11:283-91.

3. Al-Rohani M. Renal failure in Yemen. Transplant Proc. 2004 Jul-Aug;36(6):1777-9.

4. Feast TG, Rourid A, Lamad S. A prospective community based study of the incidence of severe acute renal failure in adults. BMJ. 1993;306:481-3.

5. Davenport A, Jone SR, Goel S, Astley JP, Hartog M. Differentiation of acute renal impairment by detection of carbamylated hemoglobin. Lancet. 1993;341:1614-7. 
6. Nasr E, Omar HM. Value of fingernail creatinine in differentiation of acute from chronic renal failure. African Journal of Nephrology. 1997;1:48-50.

7. Akinsola A, Arije A. Peritoneal Dialysis in Nigerian patients with a short history of advanced renal failure. West Afr J Med. 1987;6:205-9.

8. Ojogwu LI. Peritoneal dialysis in the management of hypertensive acute oliguric renal failure. Trop Geogr Med. 1983;35:385-8.

9. Akinkugbe OO. Tropical Nephropathy: an overview. Afr J Med Sci. 1992;21:3-7.

10. Lameire N, Biesen WV, Vanholder R. Acute renal failure. Lancet. 2005;365:417-30.

11. Adelekun TA, Ekwere TR, Akinsola A. The pattern of acute toxic nephropathy in Ife, Nigeria. West Afr J Med. 1999;18(1):60-3.
12. Ojogwu LI. Drug induced acute renal failure: A study of 35 cases. West Afr J of Med. 1992;11:185-9.

13. De Vriese A, Vogelaers D, Lameire N. Sepsis and acute renal failure. African Journal of Nephrology. 1997;1:12-7.

14. Kaballo BG, Khogali MS, Khalifa EH, Khaiii EA, El-Hassan AM, Abu-Aisha H. Patterns of severe acute renal failure in a referral center in Sudan: excluding intensive care and major surgery patients. Saudi J Kidney Dis Transpl. 2007 Jun;18(2):220-5.

15. Rule AD, Bergstralh EJ, Slezak JM, Bergert J, Larson TS. Glomerular filtration rate estimated by cystatin $\mathrm{C}$ among different clinical presentations. Kidney Int. 2006; 69:399.

16. Stevens LA, Levey AS. Measurement of kidney function. Med Clin North Am. 2005;89:457. 